\title{
Entanglement Detection in Coupled Particle Plasmons
}

\author{
Javier del Pino, ${ }^{1,2}$ Johannes Feist, ${ }^{1}$ F. J. García-Vidal, ${ }^{1, *}$ and Juan Jose García-Ripoll ${ }^{2}$ \\ ${ }^{1}$ Departamento de Física Teórica de la Materia Condensada and Condensed Matter Physics Center (IFIMAC), \\ Universidad Autónoma de Madrid, Madrid E-28049, Spain \\ ${ }^{2}$ Instituto de Física Fundamental, IFF-CSIC, Calle Serrano 113b, Madrid E-28006, Spain
}

(Received 27 February 2014; published 29 May 2014)

\begin{abstract}
When in close contact, plasmonic resonances interact and become strongly correlated. In this work we develop a quantum mechanical model for an array of coupled particle plasmons. This model predicts that when the coupling strength between plasmons approaches or surpasses the local dissipation, a sizable amount of entanglement is stored in the collective modes of the array. We also prove that entanglement manifests itself in far-field images of the plasmonic modes, through the statistics of the quadratures of the field, in what constitutes a novel family of entanglement witnesses. Finally, we estimate the amount of entanglement, the coupling strength and the correlation properties for a system that consists of two or more coupled nanospheres of silver, showing evidence that our predictions could be tested using present-day state-of-the-art technology.
\end{abstract}

DOI: 10.1103/PhysRevLett.112.216805

PACS numbers: 73.20.Mf, 03.67.Mn, 42.50.Dv

Surface plasmons are hybrid light-matter excitations confined at the interface between a metal and a dielectric. Due to their small mode volume and strong electromagnetic (EM) fields, surface plasmons interact very strongly with quantum optical emitters [1-4], such as quantum dots [5], NV centers [6], or inorganic [7] and organic molecules $[8,9]$. This, together with their broadband nature, small size, and inherent quantum properties, makes them a promising platform for future integrated quantum information technologies [10]. However, a very important problem lies in the characterization and control of those quantum properties. So far, several experiments have demonstrated that coupling photons in and out of plasmonic resonances preserves quantum features such as single-photon excitations and antibunching [5], photon-photon entanglement [11], energy-time entanglement [12], and squeezing [13]. In this work we focus on the quantum properties of the surface plasmon themselves and in particular on how many-body entanglement can be engineered using arrays of coupled plasmonic modes.

In this Letter, we present a plasmonic setup that intrinsically exhibits many-body entanglement and we provide a recipe for characterizing it experimentally. Our results build on a quantum mechanical model for a one-dimensional or a two-dimensional array of coupled nanoparticles [14-16] that includes the dipole-dipole interaction between particle plasmons, the losses in each nanoparticle, and the possibility of injecting energy via coherent or incoherent light. Using this model we can not only study the transport of excitations through the plasmonic band, but we also demonstrate the emergence of stationary entanglement in the array at room temperature. Moreover, we argue that this entanglement can be detected by measuring fluctuations of the light that is emitted from the plasmonic array in the far-field.
We introduce three important theoretical ideas. The first one is a quantum mechanical model for the nanoparticle array that consists of an array of coupled oscillating dipoles with nearest-neighbor interaction and a local dissipation that accounts for the losses. This model results in a master equation for the density matrix associated with the plasmonic array. The second important idea is that, under very general circumstances, this density matrix will be Gaussian [17] and all properties of the array can be deduced from expectation values or "moments" of a finite set of operators. In practice this implies a single set of exactly solvable ordinary differential equations that fully describes the evolution of the quantum surface plasmons. This technique allows us to make predictions not only on the dynamics of the dipoles (i.e., absorption and transport of energy) but also about their correlations and the resulting entanglement. Let us note that in this work we do not provide a microscopic justification for the existence of coherent degrees of freedom in the plasmonic array. Instead, our model is similar in spirit and form to the continuous variable quantum model that describes the photon-like emergent quantum degrees of freedom in superconducting circuits $[18,19]$. In this sense, the model is complete, as it is the a minimal quantum model that is consistent with the classical description and with other intrinsic properties of the plasmonic resonances, such as their linear character, their Gaussian and photonlike nature, and their local Markovian losses.

The final idea in this work is a formal study of the experimental observables that can detect the presence of entanglement in the plasmonic array, the so-called entanglement witnesses [20-24]. To this end, we study the plasmonic band and compute the fluctuations of the EM field in momentum space. We formally prove that the presence of squeezing in the light with opposite momenta is 
a signature of entanglement. From an experimental point of view, this implies that by measuring the far-field light emitted from the structure and studying its quantum fluctuations [cf. Fig. 1], the amount of entanglement that is present in the plasmonic array can be quantified. This general result is valid even when the Gaussian assumption or our underlying quantum model breaks down.

We model our coupled particle plasmons as a set of $N$ oscillating dipoles forming a linear one-dimensional array, which interact through nearest-neighbor dipole coupling and may be subject to external driving [25]. The Hamiltonian reads $(\hbar=1)$

$$
H=\sum_{n=1}^{N} \frac{\omega}{2}\left(p_{n}^{2}+x_{n}^{2}\right)+\sum_{\langle n, m\rangle} g x_{n} x_{m}+\sum_{n=1}^{N} f_{n}(t) x_{n},
$$

where $f_{n}(t)$ is a driving force, $x_{n}$ is the dipole moment of the particle plasmon and $p_{n}$ its associated canonical momentum. $g$ is the coupling strength between neighboring sites, $\langle n, m\rangle$, which are separated by a distance $\Lambda$.

We introduce local dissipation by means of a master equation to describe the evolution of the quantum state or density matrix, $\rho$. This equation groups all plasmonic losses in a single parameter, $\gamma$, which comprises all decoherence and dephasing processes due to coupling to phonons, defects, and free-space photons and/or to electron-electron scattering events, and reads

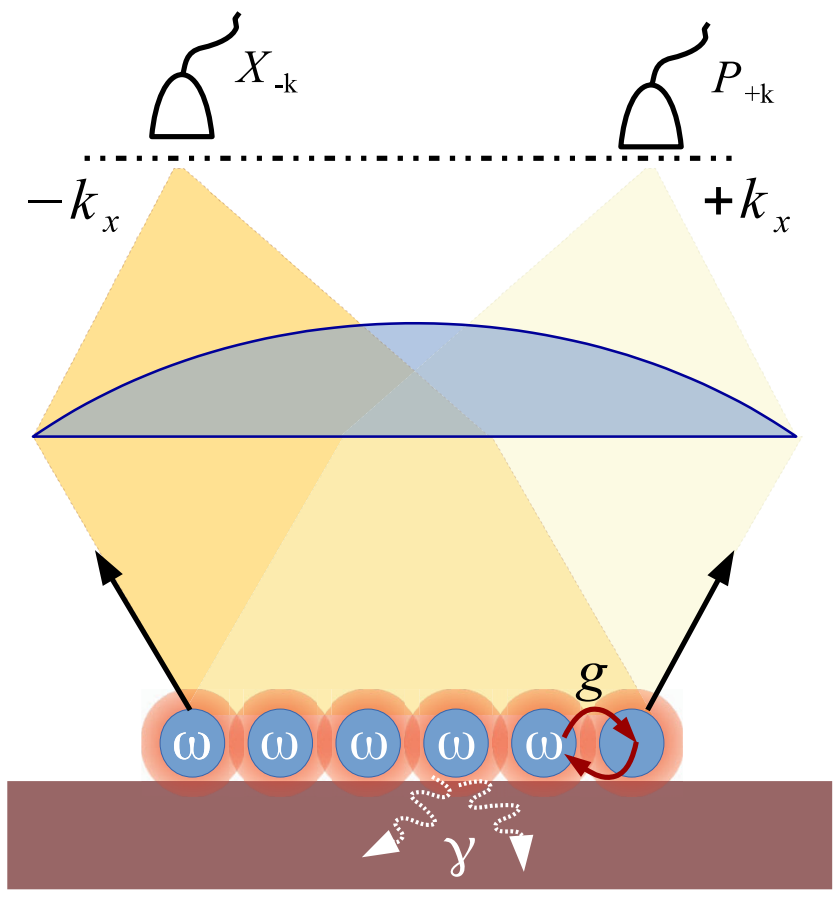

FIG. 1 (color online). An array of interacting nanoparticles gives rise to a set of coupled plasmonic modes. The far-field emission of these modes is collected by a lens. By correlating the properties of the light at different points in the focal plane, we get information about the multipartite entanglement. $\partial_{t} \rho=-\frac{i}{\hbar}[H, \rho]+\sum_{n=1}^{N} \frac{\gamma}{2}\left(2 a_{n} \rho a_{n}^{\dagger}-a_{n}^{\dagger} a_{n} \rho-\rho a_{n}^{\dagger} a_{n}\right)$,

where $a_{n}=\frac{1}{\sqrt{2}}\left(x_{n}+i p_{n}\right)$ are the Fock operators that diagonalize each individual harmonic oscillator.

Due to the quadratic nature of the problem, we can assume that the ground state of the array is Gaussian [17], as is usually done in linear optics. This implies that the density matrix $\rho$ can be reconstructed from the expectation values, $\langle O\rangle:=\operatorname{tr}(O \rho)$, of the operators $O \in\left\{x_{n}, p_{n}, x_{n} x_{m}\right.$, $\left.p_{n} p_{m}, x_{n} p_{m}\right\}$. Moreover, the evolution equations for these "moments" form a closed set of first order differential equations that can be exactly solved, as described in detail in Sec. I of the Supplemental Material [26]. Let us start with the first moment equations, which describe the dynamics of the effective dipoles $d_{n}=\left\langle x_{n}\right\rangle$. It is straightforward to find a set of coupled driven classical harmonic oscillators subject to friction,

$$
\ddot{d}_{n}=-\left(\omega^{2}+\frac{\gamma^{2}}{4}\right) d_{n}-2 \omega g \sum_{l} d_{l}-\gamma \dot{d}_{n}+\omega f_{n},
$$

where the sum over $l$ is over nearest neighbors of $n$. This is a classical model that has already been used to describe a particle plasmon array [27] and shows the compatibility of our master equation with earlier theoretical studies. In particular, our equations must describe the transport of excitations and absorption of energy by the plasmonic array. In fact, we can use the available experimental results to extract quantitative information about the three parameters $g, \omega$, and $\gamma$, which characterize our modeling.

Regarding transport, let us assume a coherent driving on the first site, $f_{1}(t) \sim \sin (\nu t)$, and study the asymptotic state of the dipoles as a function of the distance. From this calculation we can extract a propagation length, $\xi$, defined as

$$
\xi=\frac{\sum_{n=1}^{N} n \Lambda\left|\left\langle x_{n}\right\rangle\right|}{\sum_{n=1}^{N}\left|\left\langle x_{n}\right\rangle\right|} .
$$

For the case of a very long chain, this propagation length would determine the exponential decay of the plasmon population, $\left\langle x_{n}\right\rangle \sim e^{-n \Lambda / \xi}$. In Fig. 2(a) we show the propagation length in units of particle spacing, $\xi / \Lambda$, obtained numerically for a chain of $N=20$ oscillators, as a function of the coupling strength $g$ and plasmonic loss $\gamma$, under quasiresonant driving $(\nu=0.99 \omega)$. Dissipation leads to a finite propagation length, which grows with $g$ and diverges at the critical point $g / \omega=1 / 2, \gamma=0$, where the current model becomes unphysical.

While the first order moments reproduce predictions of the classical theory, the second order moments contain information about the non-classicality of the many-body particle plasmon state. In particular, the matrix of second 
order correlations, or covariance matrix, can also be exactly computed (see section I of the Supplemental Material [26]) and used to quantify the amount of entanglement present in the plasmonic array. It is given by

$$
\sigma_{i, j}=\frac{1}{2}\left\{\left\langle R_{i} R_{j}\right\rangle-\left\langle R_{i}\right\rangle\left\langle R_{j}\right\rangle\right\},
$$

where $\mathbf{R}^{T}=\left(x_{1}, \ldots, x_{L}, p_{1}, \ldots, p_{L}\right)$ is a vector that groups all positions and momenta. As is demonstrated explicitly in the Supplemental Material [26], the covariance matrix is independent of $f_{n}(t)$, whose role is merely to displace the different oscillator modes without affecting entanglement.

Let us consider a bipartition of the plasmonic array into two subarrays, $A$ and $B$. It is clear that the covariance matrix can be split into boxes that group the operators of one or the other array,

$$
\sigma=\left(\begin{array}{ll}
\sigma_{A A} & \sigma_{A B} \\
\sigma_{B A} & \sigma_{B B}
\end{array}\right)
$$

together with some off-diagonal terms, $\left\{\sigma_{A B}, \sigma_{B A}\right\}$ that imply some correlation (quantum or classical) between the two arrays. In order to quantify purely quantum correlations, we compute the so called negativity [17], $E_{N}[\sigma ; A, B]$. A value of $E_{N}[\sigma ; A, B]$ above zero means that the plasmonic array is entangled at least with respect to this bipartition. Subsequent application of this criterion to different partitions of the array can be used to ensure true multipartite entanglement.

The results of this calculation are shown in Fig. 2(b) for a one-dimensional array of 20 nanoparticles divided into two blocks of 10 consecutive particles. We plot the negativity as a function of the coupling strength $g$ and the plasmonic loss $\gamma$. We have checked that, for this range of parameters, this negativity rapidly converges to its asymptotic value for $N \rightarrow \infty$ and the results presented here for $N=20$ are indistinguishable from those obtained in that limit. As expected, entanglement grows with $g$ and becomes maximum at the critical point $g=\omega / 2, \gamma=0$, where the propagation length diverges. The effect of dissipation is to decrease the entanglement, which remains sizable for moderate coupling strengths, $g \simeq \gamma$.

Unfortunately, the negativity is not an observable. It may be estimated from the full covariance matrix if a sufficiently accurate reconstruction of this matrix is available, but this is an experimentally daunting task. It would therefore be interesting to have an experimental criterion that allows the detection of entanglement in the plasmonic chain with the least number of measurements, while being robust to noise and imperfections.

For this task we suggest what is called an entanglement witness [20-24]. A witness is an observable $W$ such that when its expectation value $\langle W\rangle=\operatorname{Tr}(W \rho)$ becomes negative, we can positively assure that the state $\rho$ is not separable. There are several such entanglement criteria in the literature of quantum optics. One of them is the socalled Duan criterion for detecting two-mode squeezing [28], which was later extended by Hyllus and Eisert [29] to include multipartite entanglement. In this work we develop a very general but simpler version of this last protocol.

Theorem: Let us take two vectors $\mathbf{u}_{1}$ and $\mathbf{u}_{2}$ which satisfy the following conditions: (i) they are normalized, $\left\|\mathbf{u}_{i}\right\|=1$, (ii) have the same modulus element-wise $\left(\left|u_{1, i}\right|=\left|u_{2, i}\right|\right)$, and (iii) they define two pairs of canonical variables,

$$
X_{k}=\sum_{j=1}^{L} u_{k, j} x_{j}, \quad \text { and } \quad P_{k}=\sum_{j=1}^{L} u_{k, j} p_{j} .
$$

If the two opposite quadratures are squeezed,

$$
\left\langle\Delta X_{1}^{2}\right\rangle+\left\langle\Delta P_{2}^{2}\right\rangle<1
$$

then the state is entangled. The demonstration of this theorem is presented in the Supplemental Material, Sec. II [26].

While the conditions (i)-(ii) might seem rather artificial, they can be satisfied by the normal modes of the plasmonic
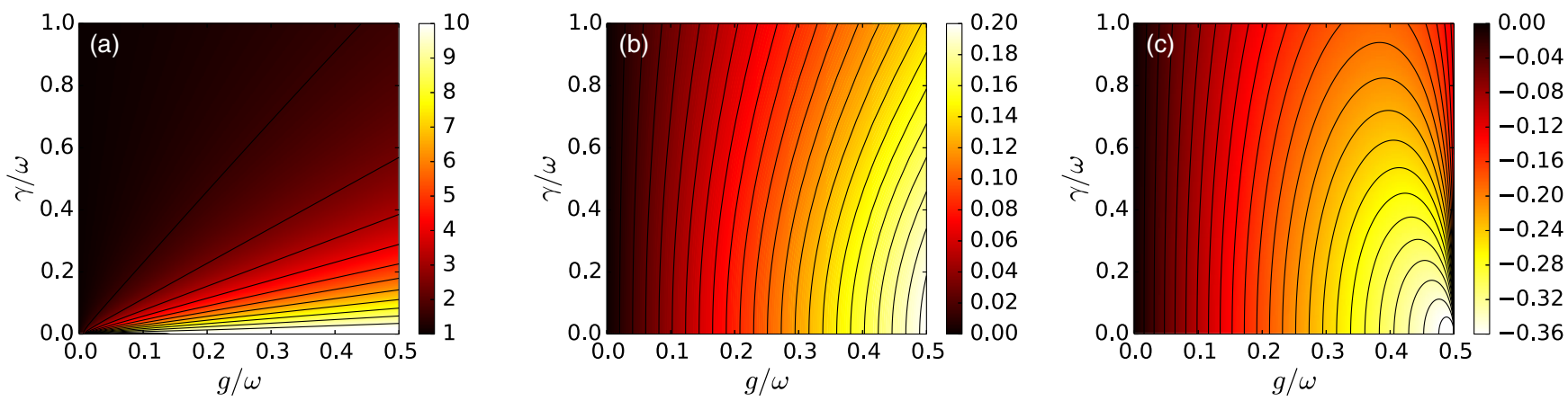

FIG. 2 (color online). (a) Average propagation length (in units of $\Lambda$ ) in the one-dimensional chain of $N=20$ nanoparticles versus coupling strength, $g$, and local dissipation, $\gamma$. (b) Entanglement in the chain measured by the logarithmic negativity. (c) Entanglement witness in momentum space. 
array. The undriven part of Hamiltonian (1) can be diagonalized using normal modes $\left\{X_{k}, P_{k}\right\}$ (see details in Sec. III of the Supplemental Material [26]),

$$
H_{0}=\sum_{k} \frac{\omega}{2}\left(P_{k}^{2}+\lambda_{k} X_{k}^{2}\right)
$$

where $k$ represents the quantized momentum, $k=$ $\pi j /[(N+1) \Lambda]$ with $j$ running from 1 to $N$. The magnitude $\lambda_{k}=1+2(g / \omega) \cos k \Lambda$ determines the plasmonic dispersion band, $\omega_{k}=\omega \sqrt{\lambda_{k}}$.

Therefore, in the case of a one-dimensional linear chain (corresponding to open boundary conditions) and for a very large number of nanoparticles, $\mathbf{u}_{1}$ and $\mathbf{u}_{2}$ of the theorem could be the wave functions associated to two eigenmodes with opposite momenta $\left(k, k^{\prime}\right)=(k, \pi / \Lambda-k)$, which are equal in modulus and only differ in the fact that one has alternating signs and the other does not, $u_{1, j}=(-1)^{j} u_{2, j}$. From a practical point of view, this means that we can detect entanglement by looking for squeezing among states with momenta $k$ and $(\pi / \Lambda-k)$. In other words, we can define our entanglement witness,

$$
W_{k}:=\min \left\{0,\left\langle\Delta X_{k}^{2}\right\rangle+\left\langle\Delta P_{\pi / \Lambda-k}^{2}\right\rangle-1\right\},
$$

so that $W_{k}<0$ implies entanglement. For the particular case $k=0$, i.e., the extrema of the dispersion band, we can find an analytical expression for the entanglement witness for $N \rightarrow \infty$ (see details in Sec. III of the Supplemental Material [26]),

$$
W_{0}=\frac{(2 g / \omega)(2 g / \omega-1)}{\gamma^{2} / \omega^{2}+4(1-2 g / \omega)}-\frac{2 g / \omega}{\gamma^{2} / \omega^{2}+4(1+2 g / \omega)} .
$$

Figure 2(c) presents the numerical results corresponding to $W_{0}$. As is shown in the plot, the growth of the witness follows the same trend as that of the negativity, hence providing the same amount of information.

In what follows we describe how this entanglement could be measured using present-day state-of-the-art technology. Squeezing in the plasmonic band is related to entanglement, and the same applies to far-field images of the lattice. The light emitted by the plasmons maps the quadratures in the collective variables $\left\{X_{k}, P_{k}\right\}$ onto the equivalent variables of the field propagating along directions $\pm k$. This light can be collected by a large aperture lens, so that each value of the momentum is mapped to a different point on the focal plane of the lens, as sketched in Fig. 1. Selecting the photons with the appropriate momenta, we can perform homodyne detection $[17,30]$ to measure the quadratures and recover the value of $W_{k}$ mentioned above. Moreover, two important features make this a very useful protocol. The first one is that our choice of witness (i.e., momentum pairs) is not relevant, as we get similar results for other values of the momentum. This is a signature that the state is indeed many-body entangled. The second one is that while we have estimated $W_{k}$ using Gaussian states, the entanglement witness is valid for any physical state. In other words, measuring $W_{k}$ detects entanglement irrespectively of the underlying physical model.

The proposed measurements could be realized using different types of coupled plasmonic modes. One interesting possibility is provided by already existing setups with gold or silver nanoparticles $[14,15]$. Earlier experiments with such nanoparticles revealed short propagation lengths, discouraging the use of such arrays for the transport of quantum information. However, in Fig. 2 it can be appreciated that, while the plasmon propagation length is related to the coupling strength and local loss, there can be a non-zero amount of entanglement even when the surface plasmons do not propagate efficiently. As an example and to provide a quantitative and realistic estimation, we have calculated the EM coupling between two silver nanospheres of radii $R=25 \mathrm{~nm}$ and separated by a distance of $2 \mathrm{~nm}$. As shown in Fig. 3, we obtain a coupling strength of around $g / \omega \approx 0.15$. From the absorption spectrum of a single nanoparticle, we can also extract a value for the loss coefficient, $\gamma / \omega \approx 0.08$. These two values for $g$ and $\gamma$ are fully compatible with earlier works studying larger arrays [31]. For this coupling and the associated plasmonic loss, we expect a measurable amount of squeezing, 12\% [see Fig. 2(c)], which would be a conclusive evidence of manybody entanglement within the plasmonic array.

Summing up, in this work we have studied a quantum model for an array of particle plasmons. The model, which

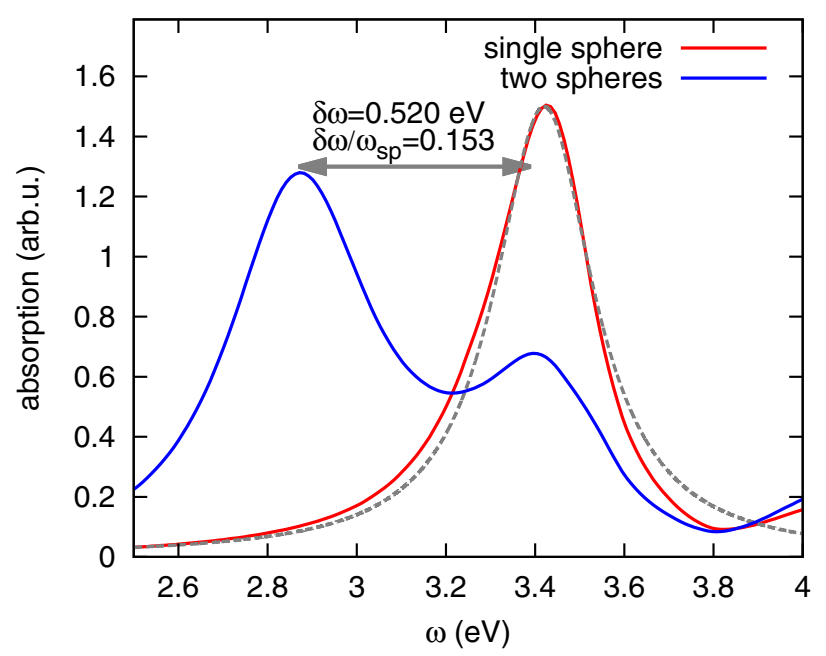

FIG. 3 (color online). Absorption versus frequency for a single silver nanosphere (red line) and a dimer (blue line). In these calculations the radii of the nanoparticles is set to $R=25 \mathrm{~nm}$ whereas the separation between nanoparticles in the dimer case is $2 \mathrm{~nm}$. The dashed grey line represents a Lorentzian fit to the absorption spectrum of the single nanosphere that is used to estimate $\gamma$. 
can be extended to any system of interacting plasmonic resonances, not only describes the collective resonances and the transport of excitations through the system, but it also predicts the existence of many-body entanglement in the system. Using the formalism of Gaussian states and entanglement witnesses we have provided an experimental protocol to detect this entanglement and estimated the strength of the measurement outcomes for realistic setups. The entanglement witness developed in this work is quite general, as it detects entanglement in far-field images even for states that are not Gaussian, including coupled surface plasmons that do not fall within our model. Moreover, some of these ideas can be exported to other fields, such as nanophotonics, matter waves, and the study of coupled resonators in superconducting circuits.

This work has been funded by the European Research Council (ERC-2011-AdG Proposal No. 290981). We also acknowledge financial support from EU FP7 project PROMISCE, CAM Research Consortium QUITEMAD (S2009-ESP-1594), and Spanish MINECO projects FIS2012-33022 and MAT2011-28581-C02-01.

*fj.garcia@uam.es

[1] D. E. Chang, A. S. Sørensen, P. R. Hemmer, and M. D. Lukin, Phys. Rev. Lett. 97, 053002 (2006).

[2] D. Dzsotjan, A. S. Sørensen, and M. Fleischhauer, Phys. Rev. B 82, 075427 (2010).

[3] M. L. Andersen, S. Stobbe, A. S. Sørensen, and P. Lodahl, Nat. Phys. 7, 215 (2011).

[4] A. Gonzalez-Tudela, D. Martin-Cano, E. Moreno, L. Martin-Moreno, C. Tejedor, and F. J. Garcia-Vidal, Phys. Rev. Lett. 106, 020501 (2011).

[5] A. V. Akimov, A. Mukherjee, C. L. Yu, D. E. Chang, A. S. Zibrov, P. R. Hemmer, H. Park, and M. D. Lukin, Nature (London) 450, 402 (2007).

[6] R. Kolesov, B. Grotz, G. Balasubramanian, R. J. Stöhr, A. A. Nicolet, P. R. Hemmer, F. Jelezko, and J. Wrachtrup, Nat. Phys. 5, 470 (2009).

[7] D. Gomez, K. Vernon, P. Mulvaney, and T. Davis, Nano Lett. 10, 274 (2010).

[8] J. Bellessa, C. Bonnand, J. C. Plenet, and J. Mugnier, Phys. Rev. Lett. 93, 036404 (2004).

[9] T. Schwartz, J. A. Hutchison, C. Genet, and T. W. Ebbesen, Phys. Rev. Lett. 106, 196405 (2011).
[10] M. Tame, K. McEnery, Ş. Özdemir, J. Lee, S. Maier, and M. Kim, Nat. Phys. 9, 329 (2013).

[11] E. Altewischer, M. Van Exter, and J. Woerdman, Nature (London) 418, 304 (2002).

[12] S. Fasel, F. Robin, E. Moreno, D. Erni, N. Gisin, and H. Zbinden, Phys. Rev. Lett. 94, 110501 (2005).

[13] A. Huck, S. Smolka, P. Lodahl, A. S. Sørensen, A. Boltasseva, J. Janousek, and U. L. Andersen, Phys. Rev. Lett. 102, 246802 (2009).

[14] S. A. Maier, M. L. Brongersma, P. G. Kik, and H. A. Atwater, Phys. Rev. B 65, 193408 (2002).

[15] S. A. Maier, P. G. Kik, and H. A. Atwater, Appl. Phys. Lett. 81, 1714 (2002).

[16] G. Weick, C. Woollacott, W. L. Barnes, O. Hess, and E. Mariani, Phys. Rev. Lett. 110, 106801 (2013).

[17] C. Weedbrook, S. Pirandola, R. García-Patrón, N. J. Cerf, T. C. Ralph, J. H. Shapiro, and S. Lloyd, Rev. Mod. Phys. 84, 621 (2012).

[18] B. Yurke and J. S. Denker, Phys. Rev. A 29, 1419 (1984).

[19] R. J. Schoelkopf and S. M. Girvin, Nature (London) 451, 664 (2008).

[20] O. Gühne and G. Tóth, Phys. Rep. 474, 1 (2009).

[21] M. Horodecki, P. Horodecki, and R. Horodecki, Phys. Lett. A 223, 1 (1996).

[22] M. Lewenstein, B. Kraus, J. I. Cirac, and P. Horodecki, Phys. Rev. A 62, 052310 (2000).

[23] B. M. Terhal, Phys. Lett. A 271, 319 (2000).

[24] D. Bruß, J. I. Cirac, P. Horodecki, F. Hulpke, B. Kraus, M. Lewenstein, and A. Sanpera, J. Mod. Opt. 49, 1399 (2002).

[25] C. Lee, M. Tame, J. Lim, and J. Lee, Phys. Rev. A 85, 063823 (2012).

[26] See Supplemental Material at http://link.aps.org/ supplemental/10.1103/PhysRevLett.112.216805 for a detailed derivation of the equations of motion, the proof of the entanglement witness, and the diagonalization of the system in terms of normal modes.

[27] M. L. Brongersma, J. W. Hartman, and H. A. Atwater, Phys. Rev. B 62, R16356 (2000).

[28] L.-M. Duan, G. Giedke, J. I. Cirac, and P. Zoller, in Quantum Information with Continuous Variables (Springer, New York, 2003), p. 145.

[29] P. Hyllus and J. Eisert, New J. Phys. 8, 51 (2006).

[30] D.-G. Welsch, W. Vogel, and T. Opatrný, in Progress in Optics, Vol. 39, edited by E. Wolf (Elsevier, New York, 1999), p. 63

[31] N. Harris, M. D. Arnold, M. G. Blaber, and M. J. Ford, J. Phys. Chem. C 113, 2784 (2009). 